\title{
Energy Spectrum of Turbulent Velocity Pulsations at Arbitrary Values of Fluid Viscosity
}

\author{
Edward V. Teodorovich \\ Ishlinsky Institute for Problems in Mechanics, Russian Academy of Sciences, Moscow, Russia \\ Email: teodor@ipmnet.ru
}

How to cite this paper: Teodorovich, E.V. (2020) Energy Spectrum of Turbulent Velocity Pulsations at Arbitrary Values of Fluid Viscosity. Journal of Modern Physics, 11, 1502-1513.

https://doi.org/10.4236/jmp.2020.1110092

Received: August 7, 2020

Accepted: October 10, 2020

Published: October 13, 2020

Copyright $\odot 2020$ by author(s) and Scientific Research Publishing Inc. This work is licensed under the Creative Commons Attribution International License (CC BY 4.0).

http://creativecommons.org/licenses/by/4.0/

\section{(c) (i) Open Access}

\begin{abstract}
The problem of calculating the energy spectrum of turbulent velocity pulsations in the case of homogeneous isotropic and stationary turbulence is considered. The domain of turbulent energy production is treated as "a black box" on which boundary the spectral energy flux is given. It is assumed that the spectrum is formatted due to intermodal interactions being local in the wave-number space that leads to a cascade mechanism of energy transfer along the wave-number spectrum and the possibility of using the renormalization-group method related to the Markovian features of the process under consideration. The obtained formula for energy spectrum is valid in a wide wave-number range and at arbitrary values of fluid viscosity. It is shown that in functional formulation of the statistical theory of turbulence, the procedure of separating local intermodal interactions, which govern energy transfer (straining effect), and filtering out nonlocal interactions, which have no influence on energy transfer (sweeping effect), is directly described without providing additional arguments or conjectures commonly used in the renormalization-group analysis of turbulent spectra.
\end{abstract}

\section{Keywords}

Spectral Flux, Spectral Energy Density, Local and Nonlocal Interactions, Inertial Term, Renormalization-Group Invariance, Epsilon-Expansion Procedure

\section{Introduction}

The problem of calculating the energy distribution over wave-numbers of turbulent fluid (spectral energy density) is a subject of numerous investigations. 
Even within the framework of the simplest model of homogeneous isotropic and stationary turbulence, solving this problem is complicated by the fact that the Navier-Stokes equations, which describe a turbulent fluid, are nonlinear and for obtaining a closed set of equations for energy one needs to express the quantity of third-order statistical moment in terms of the second-order statistical moment with the help of one or other hypotheses of closing (as a survey see [1]) or to construct a solution in the form of perturbation theory series with subsequent term-by-term averaging the series obtained [2]. In the last case, the technique of Feynman diagrams is commonly applied. The specific feature of the system under consideration consists in the fact that in a fully developed turbulence, a large number of various mode scales are excited and the effect of modes of all scales appears to be essential and has to be accounted for since a single act of intermodal interactions is only a link in a long cascade chain via the mechanism of energy transfer from the range of large-scale modes, where the turbulent energy of stochastic fluid velocity pulsations due to a development of instability of large-scale flows is produced, into the range of small-scale modes where the energy dissipates due to fluid viscosity.

Somewhat different approach to finding the spectral energy density beyond the scope of explicit applying the Navier-Stokes equations was proposed by A. N. Kolmogorov [3] who postulated that the energy transfer along the wave-number spectrum goes due to nonlinear intermodal interactions between the modes of close scales, whereas the interaction between the modes of essentially distinguished scales is realized through the cascade sequence of acts relevant to intermodal interactions between modes of intermediate scales (the Richardson-Kolmogorov cascade); in other words, it has a place "a locality in the wave-number space" of intermodal interactions that form a cascade process of energy transfer over wave-number spectrum. The question of a locality nature was discussed in detail in the surveys devoted to application of the renormalization-group method in turbulence theory [4] [5]. The locality relates to the fact that intermodal interaction between the modes with essentially different scales reduces to a primitive transfer of small-scale modes by large-scale ones without energy redistribution between modes (sweeping effect) [6]. In connection with this, when studying the spectrum form it arises the problem of selecting weak local interactions (dynamic interactions) forming the energy spectrum and acting against the ground of strong nonlocal (kinematic) interactions [7]. In the author's paper [8] it was claimed and argued the statement that in the turbulence theory with applying the RG-method [4] [5] use of the $\mathcal{E}$-expansion procedure, well-known in the theory of critical phenomena, is a way to select the local intermodal interactions and filtering out the effects of non-local (distant) interactions.

Kolmogorov proposed to divide the wave-number spectrum into three parts.

1) The range of turbulent energy production in the domain of small wave numbers $k<k_{g}$ where the energy of turbulent pulsations is generated due to 
development of instability of large-scale flows. The energy production is simulated by the action of an external random force that is similar to the Langevin force used in the theory of random processes. The energy production range lies beyond the scope of our analyses and in our model it will be treated as "a black box" when one knows nothing concerning the processes in the box and only knows conditions on its boundary (spherical surface of radius $k_{g}$ in the wave-number space). As a boundary condition it is taken the spectral flux through the boundary surface $W\left(k_{g}\right)$, which is equal to the rate of energy pumping $\mathcal{E}$ received from the range $k<k_{g}$.

2) The inertial range is a domain of wave numbers where there is no energy production and the dissipation effects are negligible. In this range the spectral flux remains constant, and it goes the process of energy transfer from the range of small wave numbers into the range of large wave numbers via the cascade sequence of local intermodal interaction acts. In the case of high Reynolds numbers (very small fluid viscosity) the inertial range has a sufficiently long length. In the inertial range it takes a place the Kolmogorov formula for spectral energy density

$$
E(k)=C_{K} \mathcal{E}^{2 / 3} k^{-5 / 3},
$$

where $C_{K}$ is the Kolmogorov constant. This formula has been obtained only on the basis of dimensionality arguments without reference to the Navier-Stokes equations.

3) The dissipation range relates to the case when the Reynolds number is not high and dissipation effects are not neglectable. As the Reynolds number decreases (the fluid viscosity grows) the domain of inertial range existence tends to zero and the Kolmogorov formula appears to be inapplicable. Below we consider the problem of building the model that is true beyond the inertial range and obtain the formula for spectral energy distribution $E(k)$ with account for viscosity that is valid for all wave-numbers with the exception of ones from the energy production range.

\section{The Problem of Calculating Energy Spectrum}

If the fluid viscosity is accounted for, the spectral flux will depend on wave number and due to the locality of intermodal interactions the spectral energy density $E(k)$ will be determined by the value of spectral flux at given wave-number $W(k)$. This fact has to be accounted for when applying the dimensionality arguments. As the result, the formula for $E(k)$ may be written in the form of "generalized Kolmogorov formula"

$$
E(k)=C(k) W(k)^{2 / 3} k^{-5 / 3}
$$

Here $C(k)$ is a dimensionless function of dimensionless variables $W(k) / W\left(k_{g}\right)$, $C\left(k_{g}\right), k / k_{g}$ and $H(k)=W(k) / v^{3} k^{4} ; v$ is the fluid viscosity and $k_{g}$ is the upper boundary of energy production range.

Beyond the energy production range $k>k_{d}$ the equation of energy balance 
has the form

$$
\frac{\mathrm{d} W(k)}{\mathrm{d} k}=-2 v k^{2} E(k)
$$

that relates two unknown quantities $W(k)$ and $E(k)$. For solving the problem one may use the energy balance Equation (2.2) in combination with the "generalized Kolmogorov formula" (2.1), but in this case it remains one more unknown function $C(k)$, which is a functional analog of the Kolmogorov constant. Thus it arises the need to have one more equation followed from some additional considerations or hypotheses. One of them was proposed by Kovazhny [9], whose conjecture appears to be equivalent to the statement $C(k)=$ const . In this approximation Equation (2.2) gives

$$
\begin{aligned}
& W(k)=\mathcal{E}\left[1+\frac{v C\left(k_{g}\right)}{2 \mathcal{E}^{1 / 3}}\left(k^{4 / 3}-k_{g}^{4 / 3}\right)\right]^{3}, \quad \mathcal{E}=W\left(k_{g}\right) \\
& E(k)=C\left(k_{g}\right) \mathcal{E}^{2 / 3}\left[1+\frac{v C\left(k_{g}\right)}{2 \mathcal{E}^{1 / 3}}\left(k^{4 / 3}-k_{g}^{4 / 3}\right)\right]^{2} k^{-5 / 3}
\end{aligned}
$$

According to Equation (2.3) the spectral flux $W(k)$ decreases as the wave-number grows, however, at a certain value of wave-number

$$
k_{d}=k_{g}\left[1+\frac{2 \mathcal{E}^{1 / 3}}{v C\left(k_{g}\right) k_{g}^{4 / 3}}\right]^{3 / 4}
$$

the flux becomes zero and at $k>k_{d}$ it becomes negative whereas the spectral energy grows that corresponds to the transport of the energy produced by a certain fictitious source from small-scale flows to large-scale ones. This "nonphysical result" points out to the fact that the approximation $C(k)=$ const $=C\left(k_{g}\right)$ is unsatisfactory and needs for a refinement. In particular, in author's paper [10] it was proposed to treat the result (2.3) as two first terms of the series expansion in fluid viscosity powers of exponent that reproduces the results obtained by Pao [11] (see also [12]). Below it will be shown that this proposal appears to be true.

\section{Cascade Mechanism of Turbulent Energy Transfer and Renormalization-Group Method}

The required additional equation, which allows one to find $C(k)$, may be obtained by taking into account the cascade mechanism of energy transfer by local in the wave-number space intermodal interactions.

The locality of intermodal interaction acts, which are treated as links of cascade chain, manifest itself in the fact that there is no a certain scale which stands out of another scales (the equal role in cascade chain of all links with given scale). In this case the characteristics of all links in cascade chain $W(k)$ and $C(k)$ are defined only by the characteristics $W_{0}=W\left(k_{0}\right), C_{0}=C\left(k_{0}\right)$ of the link with $k=k_{0}$ selected as initial one and are independent of how this link was formatted (the independence of a previous history). This means that the process 
of energy transfer over wave-number spectrum is a Markovian process. If the energy transfer be a Markovian process, we will have

$$
W(k)=W\left(k ; W_{0}, C_{0}, k_{0}\right), \quad C(k)=C\left(k ; W_{0}, C_{0}, k_{0}\right)
$$

And the dimensionality arguments enable one to represent the functions desired in the form of dimensionless functions of dimensionless variables

$$
\begin{gathered}
W(k)=W_{0} \varphi_{1}\left(k / k_{0}, H\left(k_{0}\right), C_{0}\right), \quad C(k)=C_{0} \varphi_{2}\left(k / k_{0}, H\left(k_{0}\right), C_{0}\right), \\
H(k)=W(k) / v^{3} k^{4}
\end{gathered}
$$

From Equation (3.1) it follows that the dimensionless functions $\varphi_{1,2}(x, h, c)$ of dimensionless variables $x=k / k_{0}, h=H\left(k_{0}\right)$ and $c=C_{0}$ obey the normalization conditions

$$
\varphi_{1,2}(1, h, c)=1
$$

The functions $\varphi_{1,2}(x, h, c)$ possess a certain additional symmetry related to an ambiguity in a way of setting the boundary conditions that are reduced to a choice of a certain link with wave-number $k_{0}$ as an initial link in cascade chain and specifying the characteristics of this link, namely, $W_{0}$ and $C_{0}$; in what follows the value $k_{0}$ will be referred to as the normalization point.

If another link with the wave-number $k=k_{1}$ be taken as initial one and the values of parameters of this link $W\left(k_{1}\right)=W_{1}$ and $C\left(k_{1}\right)=c_{1}$ be given, the form of functions $\varphi_{1,2}$ remains unchanged; this means that the following relationships have to be satisfied

$$
\begin{gathered}
W(k)=W_{0} \varphi_{1}\left(\frac{k}{k_{0}}, h, c\right)=W_{1} \varphi_{1}\left(\frac{k}{k_{1}}, h_{1}, c_{1}\right) \\
C(k)=c \varphi_{2}\left(\frac{k}{k_{0}}, h, c\right)=c_{1} \varphi_{2}\left(\frac{k}{k_{1}}, h_{1}, c_{1}\right)
\end{gathered}
$$

Due to the presence of ambiguity in a choice of the value $k_{0}$ (unit of scale) it follows that the functions $\varphi_{1,2}(x, h, c)$ are invariant with respect to the operation of scale transformation $k_{0} \rightarrow k_{1}$ and relevant change (renormalization) of the parameters $W_{0} \rightarrow W_{1}=W\left(k_{1}\right)$ and $c \rightarrow c_{1}=C\left(k_{1}\right)$.

The totality of above pointed operators of scale transformations in combination with renormalization of governing parameters obeys the group composition law, contains the operators of identical and inverse transformations, i.e. it made up a group called the renormalization group (RG-group), and the invariance of the function forms under RG-transformations referred to as RG-invariance. In a special case when RG-transformations and RG-invariance are related to change in putting initial or boundary conditions (our case) the term "functional self-similarity" is used [13].

Putting $k=k_{1}$ in Equation (3.3) and using the normalization condition (3.2)

$$
W_{1}=W_{0} \varphi_{1}(\alpha, h, c), \quad c_{1}=c \varphi_{2}(\alpha, h, c), \quad \alpha=k_{1} / k_{0}
$$

we arrive at the functional RG equations 


$$
\varphi_{1,2}(x, h, c)=\varphi_{1,2}(\alpha, h, c) \varphi_{1,2}(x, h, c)\left(\frac{x}{\alpha}, \frac{h \varphi_{1}(\alpha, h, c)}{\alpha^{4}}, c \varphi_{2}(\alpha, h, c)\right)
$$

here $\alpha$ is an arbitrary dimensionless parameter.

These equations are similar to the Kolmogorov-Chapman semi-group equations in the theory of Markovian random processes (the Einstein-Smolukhovsky equations in physics of Brownian motion).

By differentiating the functional RG-equations with respect to $\alpha$ and next putting $\alpha=1$ we obtain the RG-differential equations

$$
r_{1,2}(h, c) \varphi_{1,2}(x, h, c)+\left\{-x \frac{\partial}{\partial x}+\left[r_{1}(h, c)-4\right] h \frac{\partial}{\partial h}+r_{2}(h, c) c \frac{\partial}{\partial c}\right\} \varphi_{1,2}(x, h, c)=0
$$

here

$$
r_{1,2}(h, c)=\left.\frac{\partial \varphi_{1,2}(x, h, c)}{\partial x}\right|_{x=1}
$$

The functions $r_{1,2}$ are similar to the operators of infinitesimal transformations in the Lie theory of continuous groups.

In terms of the functions $\varphi_{1,2}$ the balance energy Equation (2.2) takes the form

$$
\frac{\partial \varphi_{1}(x, h, c)}{\partial x}=-2 c h^{-1 / 3} x^{1 / 3} \varphi_{1}^{2 / 3}(x, h, c) \varphi_{2}(x, h, c)
$$

from which it follows

$$
r_{1}(h, c)=-2 c h^{-1 / 3}
$$

However, the differential RG Equations (3.6) contain a new unknown quantity $r_{2}(h, c)$ determined by the function $C(k)$ in the generalized Kolmogorov Equation (2.10). Knowledge of this function is necessary for solving the RG differential equation. However, in our analyses it is enough to know this quantity in the lowest perturbation theory approximation, namely, the first-order term of a series expansion in the fluid viscosity. This is in agreement with the procedure of improving the perturbation theory by applying the RG method proposed by Bogoyubov and Shirkov in the quantum field theory [14].

To the zero-order approximation of perturbation theory it is assigned the case when $C(k)=$ const, i.e. $\varphi_{2}=1$ and $r_{2}=0$, that reproduce the Kovazhny theory containing "nonphysical singularity". The first-order approximation contains a term being proportional to fluid viscosity of first degree. An account for dimensionality arguments gives the following representation for the function $\varphi_{2}(x, h, c)$

$$
\varphi_{2}(x, h, c)=1+\mu c h^{-1 / 3}\left(x^{4 / 3}-1\right)
$$

where $\mu$ is a certain dimensionless parameter calculated by using statistical solving the Navier-Stokes equations within the framework of perturbation theory [2] at given external random force. This lies beyond the scope of the 
model under consideration. From that it follows $r_{2}(h, c)=\mu r_{1}(h, c)$ and we will analyze the relevant solution

$$
\begin{aligned}
& \varphi_{1}(x, h, c)=\left\{1-\frac{1-3 \mu}{2} c h^{-1 / 3}\left(x^{4 / 3}-1\right)\right\}^{3 /(1-3 \mu)}, \\
& \varphi_{2}(x, h, c)=\varphi_{1}^{\mu}(x, h, c)
\end{aligned}
$$

From Equation (3.10) one can see that in the range $0<\mu<1 / 3$ the solution contains "nonphysical" singularity and the position of a singular point goes from $k_{d}$ to infinity as $\mu$ goes from zero to $1 / 3$. Since the transition to $\mu \rightarrow 1 / 3$ is weakly defined, we consider this case separately by direct solving the differential RG Equation (3.6).

The equation for $\ln \varphi_{1}(x, h, c)$ can be written in the form

$$
\left\{-x \frac{\partial}{\partial x}-4 h \frac{\partial}{\partial h}+\left[r_{1}(h, c) h \frac{\partial}{\partial h}+r_{2}(h, c) c \frac{\partial}{\partial c}\right]\right\} \ln \varphi_{1}(x, h, c)=-r_{1}(h, c)
$$

From the easily verified identity

$$
\left[r_{1}(h, c) h \frac{\partial}{\partial h}+r_{2}(h, c) c \frac{\partial}{\partial c}\right] r_{1}(h, c)=\left[-r_{1}(h, c) / 3+r_{2}(h, c)\right] r_{1}(h, c)
$$

it follows that the expression in square brackets is zero when $r_{2}=r_{1} / 3$. Thus one can seek for the solution to Equation (3.10) in the form

$$
\ln \varphi_{1}(x, h, c)=r_{1}(h, c) F(x)
$$

where the function $F(x)$ obeys the equation

$$
1+\left[-x \frac{\mathrm{d}}{\mathrm{d} x}+\frac{4}{3}\right] F(x)=0, \quad F(1)=0,\left.\quad \frac{\mathrm{d} F(x)}{\mathrm{d} x}\right|_{x=1}=1
$$

that gives

$$
F(x)=\frac{3}{4}\left(x^{4 / 3}-1\right)
$$

As the result the formulas for spectral flux and spectral energy density take the forms

$$
\begin{gathered}
W(k)=\mathcal{E} \exp \left\{-\frac{3}{2} \frac{c v}{\mathcal{E}^{1 / 3}}\left(k^{4 / 3}-k_{g}^{4 / 3}\right)\right\} \\
E(k)=c \mathcal{E}^{2 / 3} k^{-5 / 3} \exp \left\{-\frac{3}{2} \frac{c v}{\mathcal{E}^{1 / 3}}\left(k^{4 / 3}-k_{g}^{4 / 3}\right)\right\}
\end{gathered}
$$

Here we choose the wave-number $k_{g}$, corresponding to the upper boundary of turbulent energy production range, as a normalization point, and use the notation $\mathcal{E}=W\left(k_{g}\right)$ and $c=C\left(k_{g}\right)$. In particular case when $k_{g}=0$ the results (3.12)-(3.13) appear to be identical to formulas proposed by Pao [11] [12], who put forward the hypothesis on proportionality of energy density $E(k)$ to spectral flux $W(k)$ without any argumentation.

If the parameter $\mu$ exceeds one third the spectral characteristics monotonically decrease as $k$ tends to infinity and no singularities arise. In this case the 
formulas for energy flux and spectral energy density take the forms

$$
\begin{gathered}
W(k)=\mathcal{E}\left\{-\frac{3}{2} \frac{c v}{\mathcal{E}^{1 / 3}}\left(k^{4 / 3}-k_{g}^{4 / 3}\right)\right\}^{-3 /(3 \mu-1)} \\
E(k)=c \mathcal{E}^{2 / 3} k^{-5 / 3}\left\{-\frac{3}{2} \frac{c v}{\mathcal{E}^{1 / 3}}\left(k^{4 / 3}-k_{g}^{4 / 3}\right)\right\}^{-(3 \mu+2) /(3 \mu-1)}
\end{gathered}
$$

\section{Local and Nonlocal Interactions}

Our analysis is based on the statement that the energy spectrum is formed due to intermodal interactions being local in the wave-number space, and this enables one to tell about a cascade mechanism of energy transfer over wave-number spectrum and a Markovian character of the process. Precisely this property of the process gives a possibility to assume that the solution describing the cascade chain depends uniquely on numerical parameters of the link in the chain treated as initial one and is independent of the fact how this link was formatted (independence on previous history). The property of independence on previous history of formatting the initial and boundary conditions (functional self-similarity) is an inherent one for differential equations that do not contain integral terms. Solutions to such equations possess the property of invariance with respect to the way of setting additional (initial and boundary) conditions. Namely, under the shift of the hyper-surface on which additional conditions are given and relevant changing (renormalization) numerical parameters specifying additional conditions the solution remains to be unchanged. The balance energy Equation (2.2) relates to the class of such equations.

However, in the statistical theory of turbulence, the chain of equations that relates statistical moments of various orders arises due to nonlinearity of the Navier-Stokes equation, and this relation necessary contains integral terms. In particular, the equation for the Fourier-transform of the second-order statistic moment of velocity field $B(k, \omega)$ has the form

$$
L^{(0)}(k, \omega) B(k, \omega)+T(k, \omega)=D^{(0)}(k, \omega) G(k, \omega)
$$

where the second-rank tensor $T(k, \omega)$, also referred to as the inertial term, is expressed via the integral of the third-order statistical moment of turbulent velocity pulsations (Equation (A.4) in Appendix). The inertial term describes the processes of momentum and energy redistribution due to mixing induced by velocity pulsations. The relevant intermodal interactions are obviously nonlocal, and the question of validity the form of balance energy Equation (2.2) and an ability to use various symmetry properties like the RG-invariance arises. Thus we arrive at the problem of filtering out these interactions. In the Yakhot-Orszag renormalization-group theory of turbulence [15], this problem is solved by using the $\varepsilon$-expansion procedure when one first calculates the quantity desired in the low-order approximation in $\varepsilon$ near the point $\varepsilon=0$ and next extends the result obtained into the point $\varepsilon=4$. This procedure is similar to the t'Hooft-Veltman dimensional regularization method in quantum field theory. 
An account for the effect of turbulent velocity pulsations leads to a correction to fluid viscosity (a turbulent viscosity), and as a result the representation for the Fourier-transform of reverse Green's function takes the form

$$
G^{-1}(k, \omega)=\left[G^{(0)}(k, \omega)\right]^{-1}-\Sigma(k, \omega)
$$

$\left[G^{(0)}(k, \omega)\right]^{-1}=L^{(0)}(k, \omega)=-i \omega+v k^{2}$ is the reverse Green function of the linear problem. (This equation can be rewritten in the form of Dyson's equation well-known in quantum-field-theory

$$
G(k, \omega)=G^{(0)}(k, \omega)+G^{(0)}(k, \omega) \Sigma(k, \omega) G(k, \omega)
$$

where $\Sigma(k, \omega)$ is referred to as the self-energy operator.)

Another result of account for a mixing by turbulent velocity pulsations consists in appearance of effective random force which variance is written as

$$
D(k, \omega)=D^{(0)}(k, \omega)+D^{(1)}(k, \omega)
$$

Here $D^{(0)}(k, \omega)$ is the variance of external random forces that simulate the emergence of stochasticity due to development of instability of large-scale flows and is similar to Langevin forces in the theory of random processes; the second summand $D^{(1)}(k, \omega)$ arises due to account for transport phenomena produced by mixing processes.

The quantity $D(k, \omega)$ enters into the equation for the second-order statistic moment of velocity field $B(k, \omega)$

$$
B(k, \omega)=G(k, \omega) D(k, \omega) G(-k,-\omega)
$$

first obtained by Schwinger [16] when building the theory of quantized fields beyond the scope of perturbation methods. The Schwinger approach is based on statistical description of quantized fields in terms of characteristic (generating) functional. In the statistical theory of turbulence this equation was obtained by Wyld [2] with the help of summing up the perturbation theory series and applying the technique of Feynman diagrams.

In the space-time variables the inertial term $T(1,2)$ contains the third-order statistical moment of velocity field (see Appendix, Equation (A4)) and the question arises whether the Fourier-transform of inertial term will contain integral terms and how to close the set of equations by excluding the third-order statistical moment.

Within the framework of statistical description of turbulence in terms of characteristic (generating) functional it can be obtained the formula for inertial term

$$
T(k, \omega)=-\Sigma(k, \omega) B(k, \omega)-G(k, \omega) D^{(1)}(k, \omega)
$$

firstly pointed out in author's paper [17]. It should be noted that this formula is an exact one since no approximations or additional conjectures were used in its derivation.

The quantities $\Sigma$ and $D^{(1)}$ are defined by solving the Navier-Stokes equations, but these quantities can be excluded by using Equation (4.2) and Equation 
(4.4) that gives

$$
T(k, \omega)=-L^{(0)}(k, \omega) B(k, \omega)+G(k, \omega) D^{(0)}(k, \omega)
$$

For the quantity $T(k)=\int T(k, \omega) \mathrm{d} \omega / 2 \pi$ we obtain

$$
T(k)=-v k^{2} B(k)+G(k) D^{(0)}(k)
$$

If to put $T(k)=\mathrm{d} W(k) / \mathrm{d} k$, we arrive at the balance energy equation that coincides with Equation (2.2) beyond the energy production range. The above presented procedure is another way of excluding nonlocal intermodal interactions when building the theory of turbulent spectra.

\section{Conclusion}

The theory of spectral energy distribution is based on the Kolmogorov conjecture that the energy spectrum is formatted by intermodal interactions being local in wave-number space. From this, it follows a cascade mechanism of energy transfer along the wave-number space, the Markovian properties of the process, as well as an ability to apply various similarity arguments such as the property of renormalization-group invariance (functional self-similarity). In this case, the problem of separating local intermodal interactions (straining effects) and filtering out nonlocal ones (sweeping effects) arises. An account for mixing processes by turbulent velocity pulsations (swimming effects) reveals in the form of an addition $D^{(1)}$ to a variance of external random forces (turbulent random force) and addition $\Sigma$ to viscous term in the Navier-Stokes equations (turbulent viscosity). In Section 4 it was shown that in the functional formulation of statistical description of turbulence, these quantities prove to be excluded from the balance energy equation. As the result, the problem of selecting local intermodal interactions and filtering out nonlocal ones appears to be solved exactly without applying other methods such as the $\mathcal{E}$-RG (see more recent survey [18]).

\section{Acknowledgements}

The work was carried out within the framework of the State Task (project No AAAA-A17-117021310375-7).

\section{Conflicts of Interest}

The author declares no conflicts of interest regarding the publication of this paper.

\section{References}

[1] Monin, A.S. and Yaglom, A.M. (1975) Statistical Fluid Mechanics, Vol. 2. MIT Press, Cambridge.

[2] Wyld, H.W. (1961) Annals of Physics, 14, 143-165. https://doi.org/10.1016/0003-4916(61)90056-2

[3] Kolmogorov, A.N. (1941) Doklady Akademii Nauk SSSR, 30, 299-303.

[4] Teodorovich, E.V. (1993) Izvestiya, Russian Academy of Sciences. Atmospheric and 
Oceanic Physics, 29, 149-163.

[5] Zhou, Y. and Vahala, G. (1992) Physical Review A, 46, 1136-1139.

https://doi.org/10.1103/PhysRevA.46.1136

[6] Kadomtsev, B.B. (1964) Plasma Turbulence. In: Problems in Theory of Plasmas, Vol. 4, Atomizdat, Moscow, 188. (In Russian)

[7] Teodorovich, E.V. (1990) Fluid Dynamics, 25, 522-527. https://doi.org/10.1007/BF01049856

[8] Zakharov, V.E. and L’vov, V.S. (1975) Izvestiya Vuzov. Radiofizika, 28, 1470-1487. https://doi.org/10.1007/BF01040337

[9] Kovasznay, L.S.G. (1948) Journal of the Aeronautical Sciences, 15, 745-753. https://doi.org/10.2514/8.11707

[10] Teodorovich, E.V. (2017) Applied Mathematics and Mechanics, 81, 450-454. https://doi.org/10.1016/j.jappmathmech.2018.03.013

[11] Pao, Y.-H. (1965) Physics of Fluids, 8, 1063-1075. https://doi.org/10.1063/1.1761356

[12] Leslie, D.C. (1973) Developments in the Theory of Turbulence. Clarendon Press, Oxford.

[13] Shirkov, D.V. (1982) Soviet Physics-Doklady, 27, 197-199.

[14] Bogolyubov, N.N. and Shirkov, D.V. (1984) Introduction to the Theory of Quantized Fields. Nauka, Moscow. (In Russian)

[15] Yakhot, V. and Orszag, S.A. (1986) Journal of Scientific Computing, 1, 3-51. https://doi.org/10.1007/BF01061452

[16] Schwinger, J. (1949) Physical Review, 75, 651-679. https://doi.org/10.1103/PhysRev.75.651

[17] Teodorovich, E.V. (2013) Journal of Modern Physics, 4, 56-63. https://doi.org/10.4236/jmp.2013.41010

[18] Zhou, Y. (2010) Physics Reports, 488, 1-49. https://doi.org/10.1016/j.physrep.2009.04.004 


\section{Appendix. Basic Equations in Space-Time Variables}

To simplify the writing in formulas we will use digital notation for space-time variables and the index of vector components $\left\{r_{1}, t_{1}, \alpha_{1}\right\} \equiv 1$ according to which $u_{\alpha_{1}}\left(r_{1}, t_{1}\right) \equiv u(1)$. Also it will be implied the integration over space-time coordinates and the summing over component indexes for coinciding digital numbers (the Einstein rule), i.e.

$$
u(1) v(1) \equiv \int u\left(r_{1}, t_{1}\right) \cdot v\left(r_{1}, t_{1}\right) \mathrm{d} r_{1} \mathrm{~d} t_{1}
$$

The Navier-Stokes equations (NSE) with the presence of the external random force $X(1)$ and the external regular force $f(1)$

$$
L^{(0)}(1,2) u(2)+\frac{1}{2} V(1 \mid 2,3) u(2) u(3)=X(1)+f(1)
$$

(for more details related to notation see [16]).

Variance of external random force

$$
D^{(0)}(1,2)=\langle X(1) X(2)\rangle
$$

The equation for $B(1,2)=\langle u(1) u(2)\rangle$

$$
L^{(0)}\left(1,1^{\prime}\right) B\left(1^{\prime}, 2\right)+T(1,2)=G\left(1,1^{\prime}\right) D^{(9)}\left(1^{\prime}, 2\right)
$$

Inertial term

$$
T(1,2)=\frac{1}{2} V(1 \mid 3,4) B(3,4,2), \quad B(1,2,3)=\langle u(1) u(2) u(3)\rangle
$$

Exact representation for inertial term

$$
T(1,2)=-B\left(1,1^{\prime}\right) \Sigma\left(1^{\prime}, 2\right)-G\left(1,1^{\prime}\right) D^{(1)}\left(1^{\prime}, 2\right)
$$

The Schwinger-Wyld formula

$$
B(1,2)=G\left(1,1^{\prime}\right) G\left(2,2^{\prime}\right) D\left(1^{\prime}, 2^{\prime}\right)
$$

$D(1,2)$ is the variance of effective random forces.

Another form of Equation (A.6)

$$
B\left(1,1^{\prime}\right) G^{-1}\left(1^{\prime}, 2\right)=G\left(1,1^{\prime}\right) D\left(1^{\prime}, 2\right)
$$

